\title{
OPTIMIZING THE SHAPE OF ROTOR BLADES FOR MAXIMUM POWER EXTRACTION IN MARINE CURRENT TURBINES
}

\author{
J.A. Esfahani and H.R. Karbasian \\ Center of Excellence on Modeling and Control Systems (CEMCS) and Mechanical \\ Engineering Department, Ferdowsi University of Mashhad, Mashhad 91775-1111, Iran \\ Phone: (+98) 915-1344361, Fax: (+98) 511-77240488 \\ Email: abolfazl@um.ac.ir, hamid.karbasian@gmail.com
}

\begin{abstract}
In this paper the shape of rotor blades in Marine Current Turbines (MCTs) are investigated. The evaluation of hydrodynamic loads on blades is performed based on the Blade Element Momentum (BEM) theory. The shape of blades is optimized according to the main parameters in the configuration and operation of these devices. The optimization is conducted based on the ability of the blades to harness the maximum energy during operating. The main parameters investigated are the tip speed ratio and angle of attack. Furthermore, the influence of these parameters on the maximum energy extraction from fluid flow over a hydrofoil is evaluated. It is shown that the effect of the angle of attack on power extraction is greater than that of the tip speed ratio, while both are found to be significant. Additionally, the proper angle of attack is the angle at which the lift to drag ratio is at its maximum value. However, if a proper angle of attack is chosen, the variations in power coefficient would not be effectively changed with small variations in the tip speed ratio.
\end{abstract}

Keywords: Marine current turbine (MCT), hydrodynamics, tidal current flows, renewable energy, optimization.

\section{INTRODUCTION}

Marine currents are formed from tidal movements and ocean circulations, and differences in temperature or salinity levels may affect the local currents (Chakrabarti and Subrata, 2005). Kinetic energy contained within marine currents can be harnessed using various technologies. The physics is similar to that of wind energy (Rourke, Boyle and Reynolds, 2009), where the power available at any site is proportional to the fluid density and the cube of its velocity (Twidell and Weir, 2006; Lee, Lu and Huang, 2009). The biggest difference between wind and marine current resources is the density of the working fluid (Bryden, Grinsted and Melville, 2004), and therefore the power output of Marine Current Energy Devices (MCEDs) is more than that of wind turbines with similar dimensions and operating conditions (Hwang, Lee and Kim, 2009). One of devices that can be used to capture energy from tidal currents is a Marine Current Turbine (MCT); which consists of a number of blades connected to a support hub which rotates around its horizontal axis. For instance, Figure 1 shows the schematic of a fullspan pitch control SeaFlow turbine with a single $11 \mathrm{~m}$ diameter rotor mounted on a steel tubular pile (Lundin and Leijon, 2010). This type of marine current device is designed for slow velocity marine currents. Its pitch control system is designed to control its angle of attack at different tip speed ratios. Furthermore, the stability of this device 
against marine storms is appreciable, and it can even produce electricity in hard atmospheric conditions.

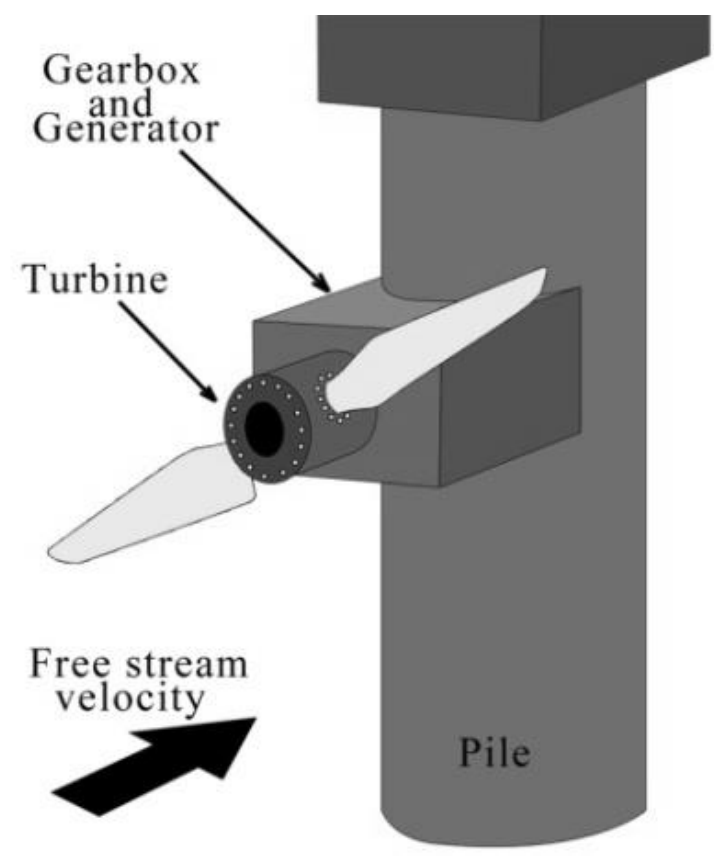

Figure 1. Schematic of Marine Current Turbine (MCT) from Schematic of Marine Current Turbines Ltd. SeaFlow project in Lynmouth, Devon, UK (Lundin and Leijon, 2010)

Many investigations have been conducted on the design horizontal MCTs that can operate under good conditions. Configurations of these turbines depend on the hydrodynamic forces generated by the fluid flow over the hydrofoil-shaped blades to generate electricity (Bryden et al, 1998). However, there is no overall agreement on the optimum shape or form of these devices (Bryden and Couch, 2006; Schonborn and Chantzidakis, 2007). The main objective of this study is to investigate hydrofoil-shaped blades optimization in order to harness the maximum energy from a fluid flow. The parameters considered in this study are the tip speed ratio (TSR) and the hydrofoil's angle of attack (AOA). According to these key parameters the optimum blade shape (chord profile along with the span of the blade) is developed. Furthermore, the sensitivity of each given parameter on the maximum energy extracted is evaluated.

\section{PHYSICAL MODEL}

Figure 2 displays a schematic of a MCT used to extract energy from tidal current flows. The root of blade (ROB) is where the blade is fixed to the hub, which is rotating the shaft. The tip of blade (TOB) is the end part of the blade and the distance from the ROB to TOB is called the turbine radius, $R$. The blade is divided into small elements, the chord and span of each element are defined as $c(r)$ and $d r$, respectively. The distance from ROB to an element of $d r$ is called the blade local radius and defined as $r$. 


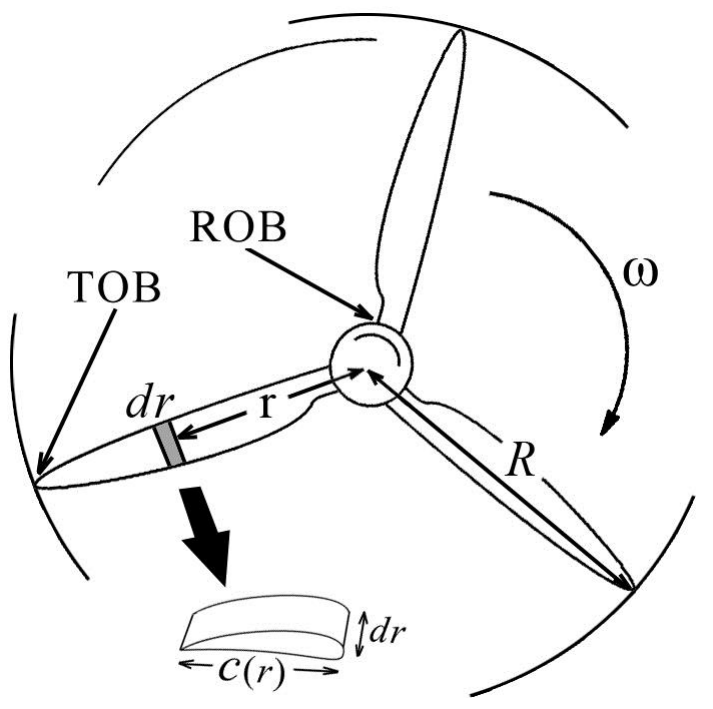

Figure 2. Schematic of MCT and preliminary concept of Blade Element Momentum

The performance of a MCT is evaluated based on Blade Element Momentum (BEM) theory. BEM theory is based on the Glauert propeller theory (Glauert, 1926), modified for application in wind turbines. In recent years, BEM theory has been optimized and modified to provide increasingly accurate results. The force acting on a blade parallel to the direction of rotation leads to the production of power, $P_{y}$, which rotates the shaft connected to the generator. $P_{y}$ is defined as follows:

$$
P_{y}=\frac{1}{2} \rho U_{0}^{3} \int_{0}^{R} c(r) \psi_{1} \lambda\left[\lambda^{2}+(1-a)^{2}\right] d r
$$

where $\rho, a, U_{0}, C_{L}, C_{D}$ are the water density, axial induction factor, free stream velocity, lift and drag coefficients of the hydrofoil, respectively. $\psi_{1}$ is defined as:

$$
\psi_{1}=C_{L} \cos \left(\tan ^{-1} \lambda\right)-C_{D} \sin \left(\tan ^{-1} \lambda\right)
$$

where $\lambda$ is also local velocity ratio and is defined as follows:

$$
\lambda=r \omega /\left[U_{0}(1-a)\right]
$$

where $\omega$ is the angular velocity of the shaft. Additionally, the TSR is given by the following relation:

$$
T S R=R \omega / U_{0}
$$

The total power available in water flow through the extraction area is given by the following relation:

$$
P_{t}=\frac{1}{2} \rho U_{0}^{3}\left(\pi R^{2}\right)
$$

The power coefficient, $C_{p}$, is defined as the ratio of kinetic power (transferred to the shaft) to the total power available:

$$
C_{P}=\frac{1}{\pi R^{2}} \int_{0}^{R} c(r) \psi_{1} \lambda\left[\lambda^{2}+(1-a)^{2}\right] d r
$$


The axial induction factor can be predicted by the procedure explained by Lanzafame and Messina (2010). In Eq. (6), all parameters are given except the chord profile of the blade (shape of the blade). To determine the maximum power extracted by MCTs, the chord profile has a significant influence on the optimization. To determine the proper chord profile it is assumed that the total power available in the water flow can be captured by the hydrofoil. Thus it can be written as follows:

where:

$$
0 \leq c(r) \leq \frac{2 \pi r}{\left[\lambda^{2}+(1-a)^{2}\right]\left(\psi_{1} \lambda+\psi_{2}\right)}
$$

$$
\psi_{2}=C_{L} \sin \left(\tan ^{-1} \lambda\right)+C_{D} \cos \left(\tan ^{-1} \lambda\right)
$$

According to the assumption in Eq. (7), the chord function is limited between zero and other parameters. The maximum chord function can capture the total power available in the water flow, however this assumption can only help determine the appropriate shape of the blades, while the Betz limit is always appreciable in the power extracted by MCTs.

\section{RESULTS AND DISCUSSION}

In this study the shape of blades employed in MCTs are designed based on maximum energy extraction from current flows. For the investigations, a NACA 63-215 hydrofoil is specified and the length of each blade (radius of turbine) is chosen as $3.5 \mathrm{~m}$. The free stream velocity is also specified as $0.5 \mathrm{~m} / \mathrm{s}$. Figure 3 shows the lift and drag coefficients of NACA 63-215 with AOA. These coefficients are experimentally determined and reported by Bertagnolio et al (2001). The AOA is one of the key parameters investigated in this study, and the importance of this parameter can be referred to as the lift to drag ratio. The lift to drag ratio determines the quantity of power generated and lost during operation, and has a great influence on the performance of MCTs.

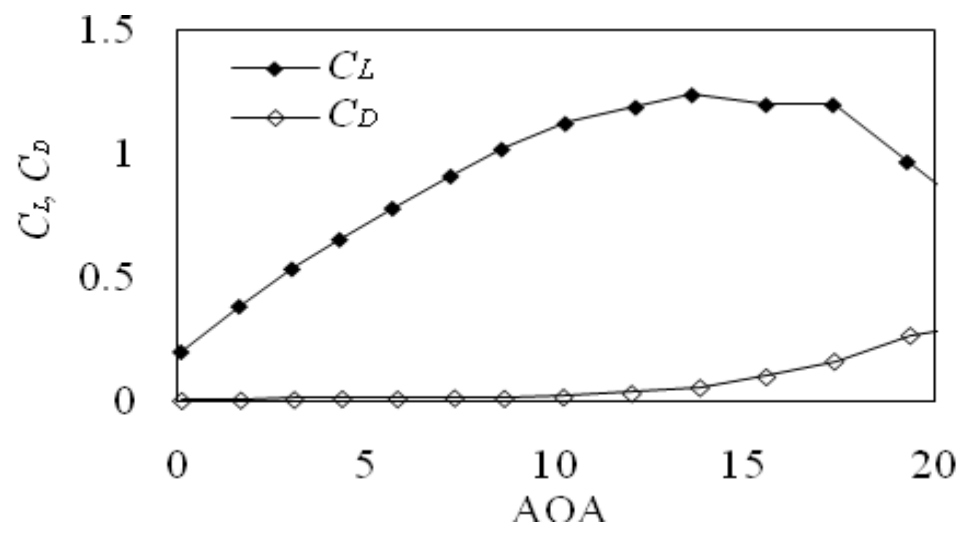

Figure 3. Experimental lift and drag coefficients for NACA 63-215, reported by Bertagnolio et al (2001)

Figure 4 displays the optimized shape of the blades for different AOA and $\mathrm{TSR}=6$. In Figure 4(a) the AOA is specified as $5^{\circ}$. At this angle the chord profile along the length of the blade is thick and has a relatively sharp edge at ROB. In Figure 4(b) 
the AOA is defined as $10^{\circ}$ and the chord profile is thinner than that of Figure 4(a). In Figure 4(c) the AOA is defined as $15^{\circ}$. At the end of the blade (TOB) the chord profile is fairly thicker than that of Figure 4(b), however at the ROB the sharp edge vanishes and variations in the chord section throughout the length of the blade become moderate.
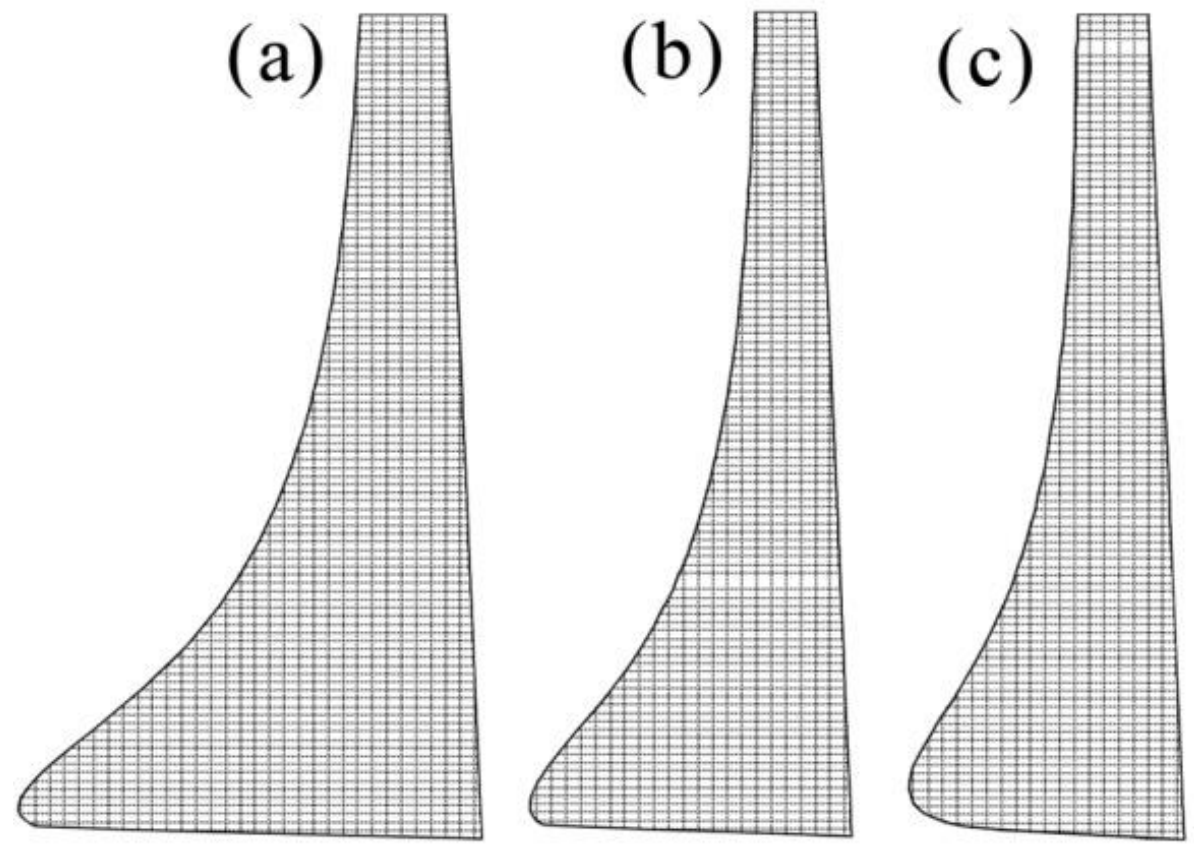

Figure 4. Optimized shape of blade at different AOAs $\left(\mathrm{AOA}=5^{\circ}, 10^{\circ}\right.$ and $\left.15^{\circ}\right)$ and $\mathrm{TSR}=6$

Figure 5 shows the optimized shape of blades for $\mathrm{TSR}=4$ and three different AOAs. In Figure 5(a) the AOA is kept at $5^{\circ}$. Thus, the variations in chord profile along the length of the blade are more pronounced and at ROB the cross-section becomes thicker. In Figure 5(b) the variations in the chord at an AOA of $10^{\circ}$ are more moderate than those of the blade with AOA of $5^{\circ}$. In Figure 5(c) the AOA is $15^{\circ}$, and all sharp variations in $\mathrm{ROB}$ and throughout the length of the blade become more moderate. The conclusions that can be drawn from Figures 4 and 5 are that the blade shape can be influenced by the TSR and AOA (lift to drag ratio). It is shown that an increasing TSR causes the optimized shape of the chord profile along the length of the blade to become thinner. Furthermore, if the AOA is kept constant throughout the length of the blade the chord becomes thicker at ROB and thinner at TOB. At ROB the sharp edge of the blade originates from the effect of the foil's drag coefficient. When the drag coefficient of the hydrofoil is increased by some manipulations in the AOA, this sharp edge would vanish and the changes in chord profile would be more moderate. Furthermore, the lift coefficient of the hydrofoil caused the changes of the chord profile to become smaller, while the overall chord profile is thinner throughout the length of the blade. 

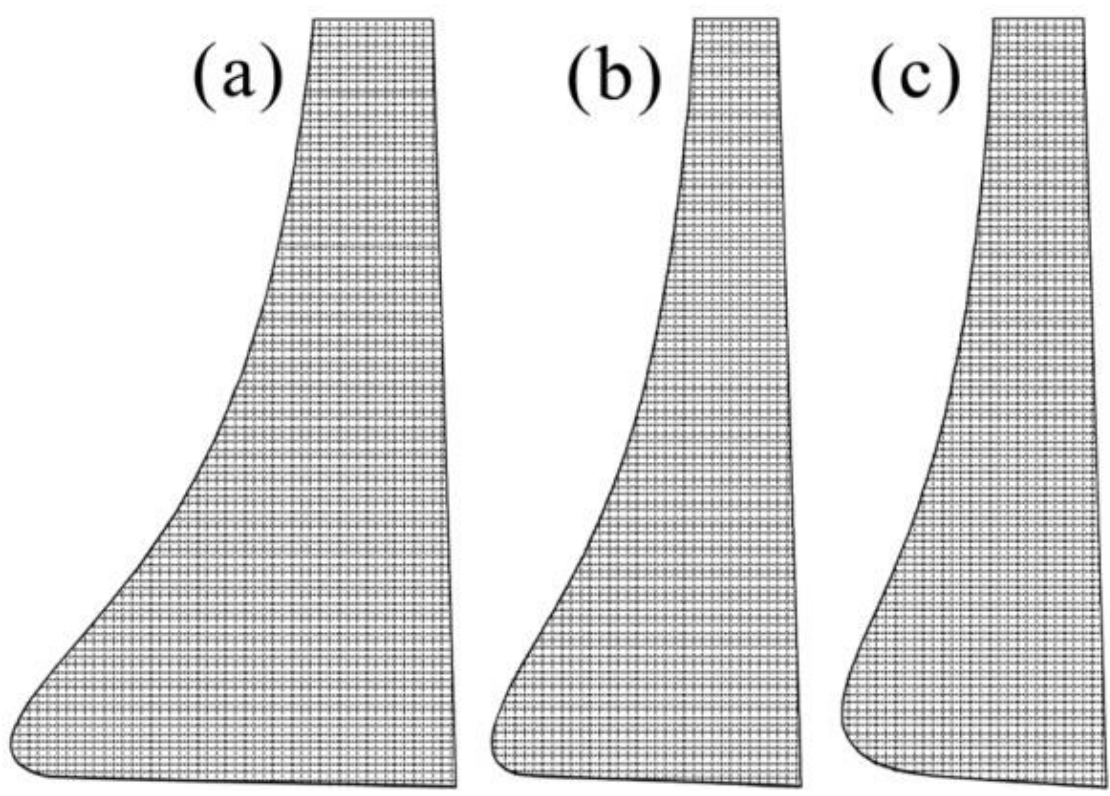

Figure 5. Optimized shape of blade at different AOA $\left(5^{\circ}, 10^{\circ}\right.$ and $\left.15^{\circ}\right)$ and $\mathrm{TSR}=4$

Figure 6 shows the variations in the partial power coefficient throughout the length of the blade. For simplicity, the length of the blade is presented as a nondimensional parameter, $r / R$, and is defined as a ratio of the blade's local radius to the radius of the turbine (R). In Figure 6, the maximum power that can be captured in each section of the blade is presented at two different AOA and TSR. As seen in Figure 6, at a constant AOA, increasing the TSR from 4 to 6 decreases the overall power coefficient. At $\mathrm{ROB}$ the power coefficient for $\mathrm{TSR}=6$ is more than that for $\mathrm{TSR}=4$. But throughout the length of the blade and reaching to TOB, the power coefficient for TSR=6 becomes smaller than that for TSR=4. Furthermore, at a constant TSR, the power coefficient for $\mathrm{AOA}=10^{\circ}$ is more than that of $\mathrm{AOA}=15^{\circ}$. This originates from the effect of the lift to drag ratio. For NACA 63-215 the lift to drag ratios are around 50 and 10 , for $A O A=10^{\circ}$ and $\mathrm{AOA}=15^{\circ}$, respectively. As seen from these ratios, a blade at $\mathrm{AOA}=10^{\circ}$, which has a higher lift to drag ratio, extracts more energy from current flows compared to a blade at $\mathrm{AOA}=15^{\circ}$. However, it is demonstrated that at $\mathrm{AOA}=10^{\circ}$ (or $\mathrm{AOA}$ at which the lift to drag ratio is at its maximum) the effect of the TSR on the power coefficient is insignificant. In other words, increasing the TSR results in the reduction in power coefficient being negligible.

Consequently, the effect of TSR and AOA on the performance of MCTs is briefly investigated and based on the results obtained with the evaluation of hydrodynamic loads acting on MCT, the best shape in the optimum range of operation is specified. As for the results, Figure 7 displays a three-dimensional blade shape which can be used at different TSRs and $\mathrm{AOA}=10^{\circ}$. This blade is designed based on a maximum lift to drag ratio beyond the static stall region. As the hydrofoil is extruded from ROB to TOB, each element of the hydrofoil is regularly twisted, so that the AOA remains constant throughout the blade's length. 


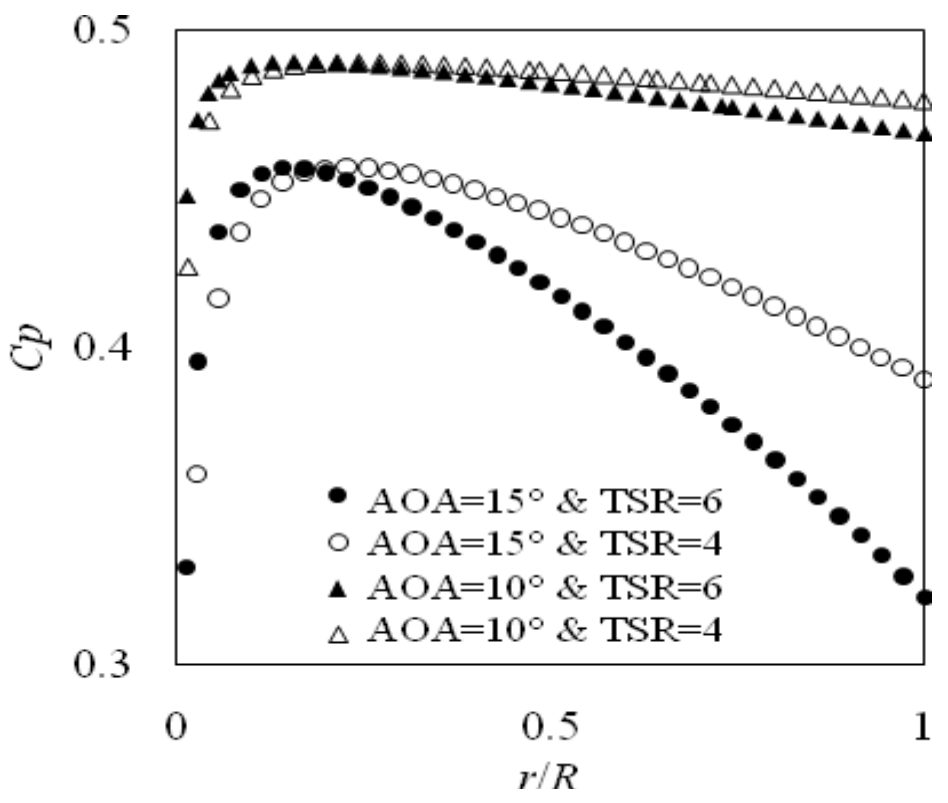

Figure 6. Partial power coefficient for each blade section at two AOA $\left(10^{\circ}\right.$ and $\left.15^{\circ}\right)$ and TSR (4 and 6)

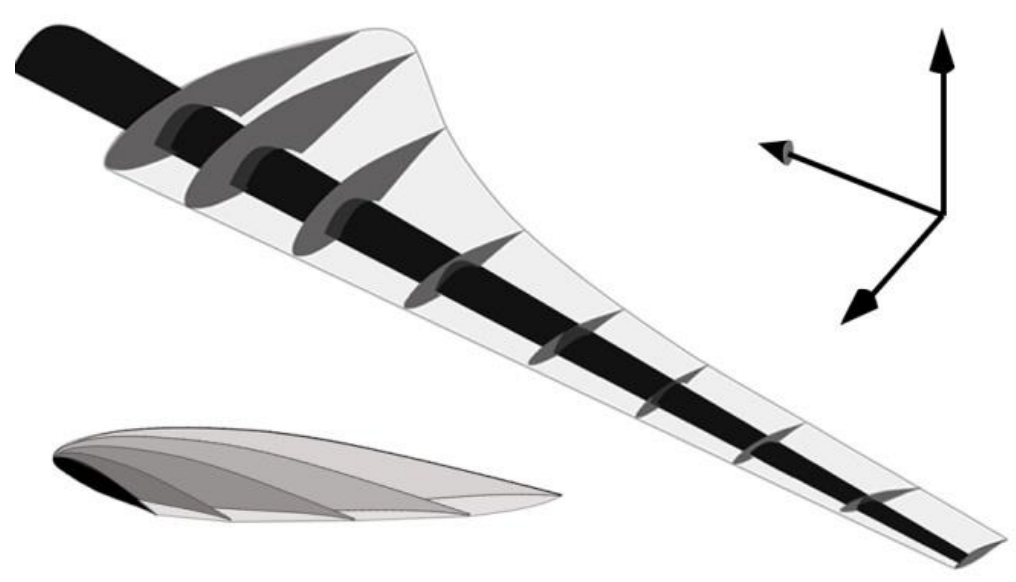

Figure 7. Three-dimensional optimized shape of blade for MCTs in optimum range of operation

\section{CONCLUSION}

The hydrodynamics of blades in MCTs are investigated based on Blade Element Momentum (BEM) theory. A NACA 63-215 hydrofoil is specified for the simulation of performance. The AOA and TSR are evaluated as key parameters in MCTs. These parameters are given values, although the shape of the blade (or chord profile) is an unknown parameter and must be determined. To determine the appropriate shape of the blade, the optimization of MCTs with a horizontal axis is considered. According to the maximum energy extracted by each blade section a chord profile is obtained. It is shown that the shape of the blades is strongly influenced by the AOA and TSR, while the influence of TSR is insignificant at higher lift to drag ratios. Additionally, it is demonstrated when the lift to drag ratio is at its maximum value, the effect of TSR on 
the shape of the blade is insignificant. Thus, the chord profile extruded thoughtout the span of the blades must be designed with a maximum lift to drag ratio at each section of the blades.

\section{ACKNOWLEDGEMENTS}

This research was supported by the Office of the Vice Chancellor for Research, Ferdowsi University of Mashhad, under Grant No. 22752.

\section{REFERENCE}

Bertagnolio, F., Sorensen, N., Johansen, J. and Fuglsang, P. 2001. Wind turbine airfoil catalogue. Roskilde, Ris $\varnothing$ National Laboratory.

Bryden, I. and Couch, S. 2006. Marine energy extraction: tidal resource analysis. Renewable Energy, 31(2): 133-139.

Bryden, I., Grinsted, T. and Melville, G. 2004. Assessing the potential of a simple tidal channel to deliver useful energy. Applied Ocean Research, 26(5): 198-204.

Bryden, I., Naik, S., Fraenkel, P. and Bullen, C. 1998. Matching tidal current plants to local flow conditions. Energy, 23(9): 699-709.

Chakrabarti, S. and Subrata, K. 2005. Ocean environment, Handbook of offshore engineering. London, Elsevier.

Glauert, H. 1926. The elements of airfoil and airscrew theory. Cambridge: Cambridge University Press.

Hwang, I., Lee, Y. and Kim, S. 2009. Optimization of cycloidal water turbine and the performance improvement by individual blade control. Applied Energy, 86(9): $1532-1540$.

Lanzafame, R. and Messina, M. 2010. Horizontal axis wind turbine working at maximum power coefficient continuously. Renewable Energy, 35(2): 301-306.

Lee, M., Lu, C. and Huang, H. 2009. Reliability and cost analyses of electricity collection systems of a marine current farm - a Taiwanese case study. Renewable and Sustainable Energy Reviews, 13(8): 2012-2021.

Lundin, M. and Leijon, M. 2010. Ocean energy. [Online] Available from: http://www.eusustel.be [Accessed 26 August 2010].

Rourke, F., Boyle, F. and Reynolds, A. (2009) Renewable energy resources and technologies applicable to Ireland. Renewable and Sustainable Energy Reviews, 13(8): 1975-1984.

Schonborn, A. and Chantzidakis, M. 2007.Development of a hydraulic control mechanism for cyclic pitch marine current turbines. Renewable Energy, 32(4): 662-679.

Twidell, J. and Weir, T. 2006. Renewable energy resources. United Kingdom: Taylor and Francis. 